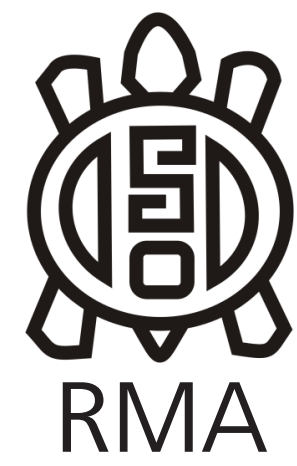

Dossier

\title{
Análisis de una cantera-taller de madera silicificada en el extremo-sur del Macizo del Deseado (Patagonia, Argentina)
}

\author{
Analysis of a silicified wood quarry at the Southern end of the Deseado \\ Massif (Patagonia, Argentina) \\ Lucas Vetrisano*, Nora V. Franco** y Pablo E. Bianchi*** \\ * Instituto Multidisciplinario de Historia y Ciencias Humanas, CONICET, \\ Argentina. E-mail: lucasvetri@yahoo.com.ar \\ ** Instituto Multidisciplinario de Historia y Ciencias Humanas, CONICET, Facultad de \\ Filosofía y Letras, Universidad de Buenos Aires, Argentina. \\ E-mail: nvfranco2008@gmail.com \\ *** Instituto Multidisciplinario de Historia y Ciencias Humanas, \\ Agencia Nacional de Promoción Científica y Técnica, Argentina. \\ E-mail: bianchipabloem@gmail.com
}

\begin{abstract}
Resumen
El Macizo del Deseado es un distrito geológico que presenta una alta disponibilidad de materias primas de excelente calidad para la talla. En nuestra área de estudio, en el extremo sur del Macizo, las formaciones Baqueró y Bajo Grande presentan fuentes primarias y secundarias de madera silicificada, pero de distribución acotada y escasa visibilidad. Diversos estudios en el área mostraron la existencia de variabilidad respecto de las características macroscópicas, el grado de silicificación y la localización de la madera silicificada. Las primeras evidencias de utilización de esta materia prima se registran hacia el 8000 A.P., en un sector del espacio en que se encuentra inmediatamente disponible, si bien las primeras ocupaciones humanas datan de la transición Pleistoceno-Holoceno. En este trabajo se presenta el análisis de una de estas fuentes primarias con el objetivo de evaluar si las características particulares de esta materia prima condicionaron de alguna manera su aprovechamiento por parte de los grupos cazadores recolectores.
\end{abstract}

Palabras clave: Cazadores recolectores; Tecnología lítica; Cantera-taller; Madera silicificada; Patagonia.

\begin{abstract}
The Deseado Massif is a geological district that presents a high availability of excellent knapping quality raw materials. In our study area, at the Southern end of the Massif, the Baqueró and Bajo Grande Formations, present primary and secondary sources of silicified wood, but with a limited distribution and poor visibility. Several studies in the area showed the existence of variability with respect to the macroscopic characteristics, degree of silicification and location of the silicified woods. The first evidence of the use of this raw material is recorded around 8000 B.P., in a space where it is immediately available, although the first human occupations of the area date from the PleistoceneHolocene transition. This paper presents the analysis of one of these primary sources with the aim of evaluating if the particular characteristics of this raw material conditioned in some way its use by the hunter-gatherer groups.
\end{abstract}

Keywords: Hunter-gatherers; Lithic technology; Quarry; Silicified wood; Patagonia.

Este trabajo se focaliza en el sur del Macizo del Deseado, provincia geológica conocida por la abundancia de rocas de excelente calidad para la talla (entre otros, Cattáneo 2000, 2004, Franco et al. 2015, Hermo 2008, Ramos 1999, Skarbun 2009). A pesar de su amplia disponibilidad, estas materias primas tienen una distribución espacial heterogénea, al igual que en otros sectores del Macizo (entre otros, Franco et al. 2015, Hermo 2008). Estas rocas, que en nuestra área de estudio provienen mayormente de rellenos silíceos de origen hidrotermal, pertenecen a las formaciones Chön Aike, La Matilde, Bajo Grande y Baqueró (e.g., Echeveste 2005, Panza y Haller 2002, Panza y Marin 1998, Zubia 1998).
El extremo sur del Macizo del Deseado presenta ocupaciones humanas discontinuas entre hace $c a$. 10.845 y 290 años A.P. (Aguerre 2003, Durán et al. 2003, Franco et al. 2010, 2013, 2019). Al igual que en espacios ubicados más hacia el norte en el Macizo, se han identificado períodos de abandono de este espacio y cambios tecnológicos que en algunos casos suceden a estos períodos de abandono (entre otros, Brook et al. 2013, Mosquera 2016). Entre los cambios tecnológicos, cabe mencionar un aumento en la frecuencia de hojas laminares hacia los 4500 años A.P. (Aguerre 2003, Franco et al. 2016), lo que está en concordancia con lo registrado a escala supra-regional (Gradin et al. 1979). 
Figura 1. Mapa del área de estudio, ubicación de los sitios mencionados en el trabajo y detalle del sector ocupado por la cantera-taller. Referencias: 1. La Gruta 3; 2. La Gruta 1; 3. El Verano; 4. Viuda Quenzana 7: 5. Viuda Quenzana 8; 6. La Martita Cueva 4.

Figure 1. Map of the study area with the location of the sites mentioned in the paper and a detail of the area filled by the quarry. References: 1. La Gruta 3; 2. La Gruta 1; 3. EI Verano; 4. Viuda Quenzana 7: 5. Viuda Quenzana 8; 6. La Martita Cueva 4

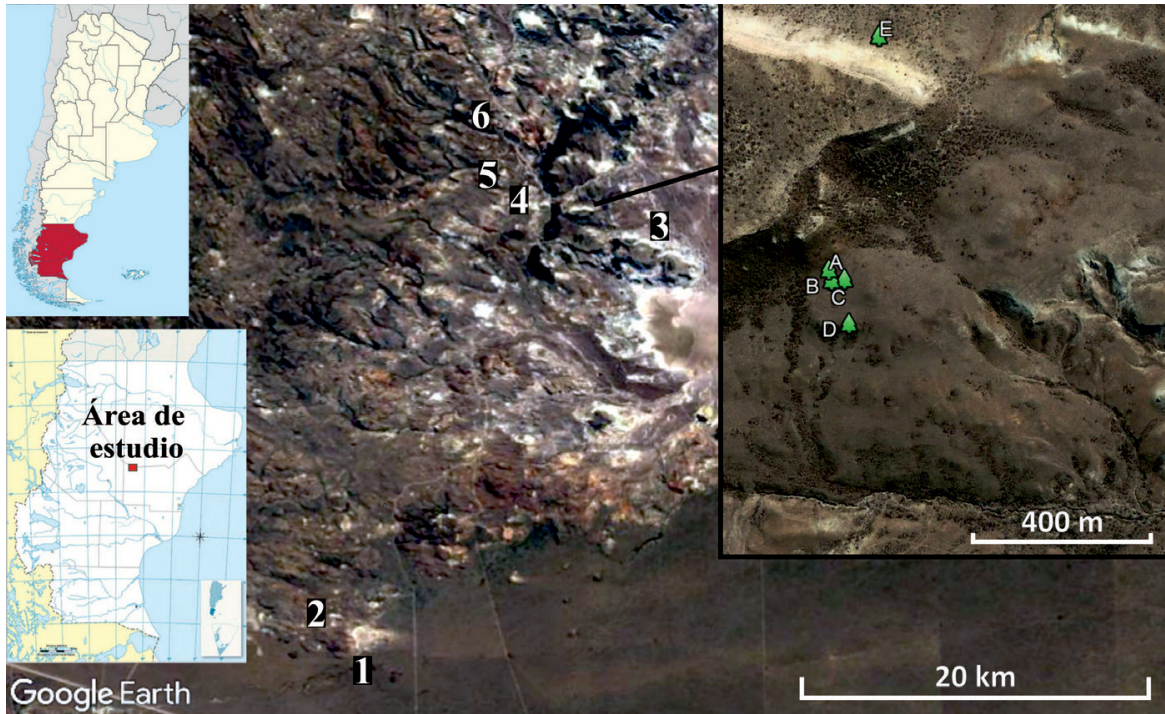

El aprovechamiento de la madera silicificada está presente en el registro arqueológico de nuestra área de estudio desde al menos el Holoceno temprano, tanto en La Gruta 1 como en la cueva 1 de El Verano (Durán et al. 2003, Franco et al 2010, 2013). Por otra parte, en áreas del Macizo ubicadas más hacia el norte, la disponibilidad de esta materia prima es mayor (por ejemplo, Cattáneo 2005a y b, Frank et al. 2013, Hermo 2005) y está presente en baja frecuencia en sitios arqueológicos desde la transición Pleistoceno-Holoceno. Este es el caso, por ejemplo, de los sitios Casa del Minero, Cueva Túnel (entre otros, Frank et al. 2013, Skarbun et al. 2007), Cerro Tres Tetas (por ejemplo, Paunero 2000, 2003) y, más hacia el norte, Piedra Museo (entre otros, Cattáneo 2005a y b, Miotti y Cattáneo 2003).

En el extremo sur del Macizo del Deseado, la madera silicificada o xilópalo tiene una distribución acotada en el paisaje, presentándose en mayor frecuencia hacia el norte de nuestra área de estudio. En nuestro caso, corresponde a las formaciones Bajo Grande (Jurásico Superior) y Baqueró (Cretácico Inferior) y presenta gran variabilidad en sus características macroscópicas, su grado de silicificación y su posición topográfica, en distancias inferiores a $10 \mathrm{~km}$ (Figura 1). Dadas las particulares características del xilópalo, principalmente la existencia de variaciones en el grado de silicificación y la presencia de vetas que pueden funcionar como planos de debilidad que afecten su fractura (ver por ejemplo Frank et al. 2013), se considera posible que, en los casos en que esta materia prima no alcanzara la calidad adecuada, esto condicionara su aprovechamiento por parte de los cazadores-recolectores. En este sentido, ya en la década de 1970, Crabtree (1972) señaló la importancia de que la materia prima para tallar sea homogénea y sin granos que la conviertan en inadecuada en proporción al grado de debilidad. Posteriormente, distintos investigadores (por ejemplo, Callahan 1979, Manninen y Knutsson 2014, Nami 1986, Odell 2004, Whittaker 1994) han sostenido la importancia de la calidad de la materia prima en la selección efectuada por los talladores. Esta calidad, de acuerdo con su experiencia como talladores se basaba en la homogeneidad de las rocas, la existencia de impurezas, fisuras, alteraciones y el tamaño de los granos (entre otros, Callahan 1979, Crabtree 1972, Inizan et al. 1992, Nami 1986). Andrefsky (1994) ha defendido, por ejemplo, que la calidad de la materia prima jugaba un papel directo en las decisiones de los grupos humanos de producir distinto tipo de artefactos. Si bien es cierto que las diferencias en las materias primas pueden afectar la morfología de los artefactos, cada caso debe ser analizado en particular ya que otros factores no relacionados con la calidad de la materia prima pueden ser responsables de estas diferencias (por ejemplo, Eren et al. 2014).

El objetivo de este trabajo es evaluar, a partir del análisis de una muestra artefactual proveniente de una fuente primaria de madera silicificada, si sus características particulares condicionaron la forma en que ésta fue aprovechada en la cantera-taller. Esta posibilidad se evalúa observando los métodos y técnicas involucrados en el proceso de reducción lítica, con especial énfasis en sus primeras etapas. Se entiende que la pregunta es relevante debido a la presencia en el registro arqueológico del área de métodos de desbaste laminar y a su posible vinculación con la madera silicificada. Esto radica en que si existe algún tipo de condicionamiento para el desbaste que obligue a realizar las extracciones siguiendo el sentido de las vetas, esto conllevaría la reducción de volúmenes prismáticos con extracciones paralelas provenientes de una o dos plataformas opuestas. La proliferación de aristas que guíen las extracciones, como resultado de su paralelismo, puede permitir que los productos del desbaste alcancen proporciones laminares.

\section{Localización y características generales de la cantera- taller analizada}

La cantera-taller analizada está localizada en una pendiente que se eleva hacia el noroeste, hasta culminar 
Figura 2. Vista de la cantera-taller, incluidos los troncos y artefactos en superficie.

Figure 2. View of the quarry, including the logs and the surface artefacts.

en una pequeña meseta. El sector de la pendiente en la que se encuentra es relativamente plano, ubicado en una cota de entre 325 y 340 m, con una inclinación promedio del 10,6\% con respecto al arroyo próximo. Debido a la pendiente del terreno la cobertura vegetal es limitada y la visibilidad de los artefactos en superficie es muy buena. La fuente primaria está compuesta por cuatro troncos (ejemplares A, B, C y D), cuyas dimensiones oscilan aproximadamente entre los 500 y $200 \mathrm{~cm}$ de largo y entre 100 y $20 \mathrm{~cm}$ de diámetro. Todos los troncos están naturalmente fracturados, mayormente in situ, pero además presentan lascados en variadas frecuencias y dimensiones (Figura 2). La dispersión total de la canterataller, considerando a los cuatro troncos, los fragmentos naturales y los artefactos, alcanza los 0,03 km². Los troncos $A, B$ y $C$ se encuentran muy próximos, a una distancia aproximada de entre 22 a $27 \mathrm{~m}$ entre sí. La mayoría de los artefactos y fragmentos se concentra en torno a estos tres troncos, en una superficie que cubre aproximadamente $3200 \mathrm{~m}^{2}$. En la parte más baja de esta misma pendiente se encuentra el tronco $D$, a una distancia de $90 \mathrm{~m}$ respecto del resto, mientras que en el sector próximo al arroyo, a más de $250 \mathrm{~m}$ de distanciase identificaron también varios fragmentos de troncos de menor tamaño. Además, a $500 \mathrm{~m}$ de la fuente, en la ladera norte de la meseta se ubican otros fragmentos de troncos, (agrupados en la Figura 1 como E) también de inferior calidad. Cabe señalar que los troncos no son visibles desde espacios cercanos más altos o bajos, como sería el caso del arroyo, y que es necesario estar localizados casi sobre ellos para observarlos.

\section{Metodología}

A los efectos de cumplir con el objetivo, se realizó una transecta de recolección que atravesó la dispersión del material de la cantera, considerando además la pendiente del terreno y la presencia de escorrentías. La transecta inició en el sector más bajo y próximo al arroyo, en dirección al sector lindante con los troncos $A, B$ y $C$, cubriendo un área de $400 \mathrm{~m}$ de largo por $15 \mathrm{~m}$ de ancho que fue cubierta por cuatro operadores. El trazado quedó paralelo a los cursos de escorrentía que desembocan en el arroyo. La recolección incluyó a todos los artefactos presentes en esta superficie, tanto instrumentos como desechos de talla, incluyendo núcleos dentro de estos últimos. No se removieron fragmentos de madera silicificada que no tuvieran evidencias de modificación antrópica.

Debido al interés en analizar si la presencia de vetas condicionaba la forma de reducción de esta materia prima, fue necesario evaluar si el eje técnico (sensu Aschero 1983) de las formas base era paralelo o transversal a las vetas. Por otra parte, se consideró importante analizar si existieron variaciones en los métodos de talla utilizados que pudieran vincularse con el grado de silicificación, el que a su vez se relaciona con la calidad de materia prima. En este sentido, para evitar dañar a los troncos, su grado de silicificación y su calidad para la talla se evaluaron macroscópicamente en el campo. Otro factor que se consideró en relación con la calidad fue la presencia de defectos, asociados a la falta de homogeneidad de la madera silicificada, entre los que se incluyen los planos de debilidad y las fisuras asociados a las vetas, los astillamientos y las fracturas en ángulos rectos. A continuación se detallan las categorías resultantes:

- Grado 0: fractura no concoidea; calidad regular

- Grado 1: fractura concoidea guiada por las vetas; calidad buena

- Grado 2: fractura concoidea condicionada por defectos; calidad que oscila entre buena y muy buena

- Grado 3: fractura completamente concoidea (sin defectos); calidad que oscila entre muy buena y excelente.

El análisis de los artefactos se realizó siguiendo los criterios delineados por Aschero (1975, 1983). Dado que la fragmentación puede aumentar el tamaño de la muestra de algunas categorías, para evaluar el paralelismo o transversalidad de las extracciones en relación con la dirección de las vetas y las formas base obtenidas, en primer lugar se presenta la información para el total de la muestra y en segundo lugar, para los instrumentos, artefactos enteros y fragmentos proximales de artefactos. 
Tabla 1. Presencia o ausencia de paralelismo entre el eje técnico de las extracciones y el sentido de las vetas de la madera silicificada. Las frecuencias se dividen según el grado de silicificación de la materia prima, considerando primero el total de los artefactos y luego, aquéllos enteros y fragmentos proximales, a efectos de no incrementar el tamaño de la muestra.

Table 1. Presence or absence of parallelism between the knapping axis and the direction of the veins in the silicified wood. The frequencies are divided according to the degree of silicification of the raw material, first considering the total of the artefacts and then only the complete ones and the proximal fragments, in order not to increase the sample size.

\begin{tabular}{|c|c|c|c|c|c|c|c|c|c|}
\cline { 3 - 10 } \multicolumn{2}{c|}{} & \multicolumn{2}{c|}{ Grado 0 } & \multicolumn{2}{c|}{ Grado 1 } & \multicolumn{2}{c|}{ Grado 2 } & \multicolumn{2}{c|}{ Grado 3 } \\
\cline { 3 - 10 } & Paralelas & 0 & 0 & 3 & 100 & 164 & 57.75 & 17 & 43.59 \\
\hline \multirow{4}{*}{\begin{tabular}{c} 
Total artefactos \\
\cline { 2 - 11 }
\end{tabular}} & Transversales & 0 & 0 & 0 & 0 & 85 & 29.93 & 19 & 48.72 \\
\cline { 2 - 11 } & N/C & 0 & 0 & 0 & 0 & 35 & 12.32 & 3 & 7.69 \\
\cline { 2 - 11 } & Total & 0 & 0 & 3 & 100 & 284 & 100 & 39 & 100 \\
\hline \multirow{3}{*}{$\begin{array}{c}\text { Total artefactos } \\
\text { enteros y fg. } \\
\text { proximales }\end{array}$} & Paralelas & 0 & 0 & 0 & 0 & 102 & 53.4 & 12 & 40 \\
\cline { 2 - 11 } & Transversales & 0 & 0 & 0 & 0 & 74 & 38.74 & 17 & 56.66 \\
\cline { 2 - 11 } & N/C & 0 & 0 & 0 & 0 & 15 & 7.85 & 1 & 3.34 \\
\cline { 2 - 11 } & Total & 0 & 0 & 0 & 0 & 191 & 100 & 30 & 100 \\
\hline
\end{tabular}

Las materias primas se identificaron por comparación macroscópica con muestras analizadas previamente por el Dr. E. Aragón (CIG-CONICET), sea a ojo desnudo y/o mediante cortes delgados.

\section{Resultados}

Los troncos que conforman la fuente no son homogéneos en cuanto a su calidad para la talla, debido al variado grado de silicificación que presentan. No es posible cuantificar con precisión la cantidad de material correspondiente a cada grado en los troncos, debido a que la corteza no es diagnostica por su bajo grado de silicificación, la cual varía de forma progresiva a lo largo de los troncos. A pesar de estas dificultades, se pueden realizar algunas observaciones generales. Los tres troncos más cercanos a la transecta (ejemplares A, B y C) son de mejor calidad y están compuestos mayoritariamente por porciones de grado 2, con algunos sectores de grado 3. El ejemplar restante (D), más alejado de la transecta de recolección, está compuesto íntegramente por materia prima del grado 2, mientras que los fragmentos hallados próximos al arroyo y los ubicados al norte de la meseta (ejemplar E) son de grado 1. Como se mencionó antes, todos los troncos tienen sectores de menor calidad vinculados a la corteza, de espesor variable. En términos generales la corteza presenta un menor grado de silicificación, que implica una textura gruesa con vetas que actúan como planos de debilidad, dificultando la dispersión isotrópica de la fuerza y, por consiguiente, limitando el control del desbaste.

La muestra recolectada en la transecta está compuesta por 338 artefactos, siendo la densidad artefactual de 0,01 artefactos por $\mathrm{m}^{2}$. El 96,32\% corresponde a madera silicificada. Las restantes materias primas son ópalos, sílices y pelita silicificada.
La mayor parte de la muestra está conformada por madera del grado $2(87,12 \%)$, seguida por los artefactos de grado $3(11,96 \%)$ y de grado $1(0,92 \%)$ (Tabla 1$)$. No se recuperaron artefactos de grado 0 . A pesar de que para los casos de los grados 1 y 2 -tanto en lo que respecta al total de los artefactos como para aquéllos enteros y los fragmentos proximales-, la frecuencia de extracciones paralelas a las vetas supera a las transversales (100\% en el caso del grado 1, mientras que en el grado 2 es de $57,75 \%$ contra $29,93 \%$ si se considera el total de artefactos y $53,4 \%$ contra $38,74 \%$ si se consideran sólo los artefactos con talón), el número de estas últimas es alto. Aunque la muestra es pequeña, esto puede implicar que si bien es posible extraer lascas atravesando el sentido de las vetas, esto podría acarrear un menor control del desbaste, lo que resultaría en una preferencia técnica por el desbaste paralelo a las vetas. En el caso del grado 3, la cantidad de extracciones transversales es mayor que la de las paralelas $(43,59 \%$ contra $48,72 \%$ si se considera el total de artefactos y $40 \%$ contra $56,66 \%$ si se consideran sólo los artefactos con talón); esto es consistente con el hecho de que la alta calidad de la materia prima no acarrea ninguna restricción relativa al proceso de reducción lítico, permitiendo la expansión isotrópica de la fuerza del golpe. En la muestra predominan los desechos de talla, que llegan a constituir el total de los artefactos del grado 1 (Tabla 2). Todos los instrumentos recuperados (ocho en total) corresponden al grado 2 y el $40 \%$ está fragmentado. Predominan los instrumentos unifaciales (raederas, artefactos mediano-pequeños en bisel asimétrico oblicuo -RBO- y muescas), aunque se recuperó un artefacto bifacial y un fragmento no diferenciado de artefacto formatizado confeccionado sobre un artefacto bifacial. La falta de instrumentos de grado 3 podría relacionarse con el traslado de la materia prima por su buena calidad hacia otros sectores del espacio, lo que deberá ser analizado en relación con los hallazgos que se hagan en sitios cercanos. Los desechos correspondientes al grado 1 incluyen dos 
Tabla 2. Categorías artefactuales divididas según el grado de silicificación de la materia prima. Los instrumentos se subdividen según el grupo tipológico y los desechos según la forma base. En primer término se considera el total de los artefactos y luego, aquéllos enteros y fragmentos proximales, a efectos de no incrementar el tamaño de la muestra.

Table 2. Artefact categories divided according to the degree of silicification of the raw material. The tools are typologically subdivided according to their retouch and the waste according to the type of flake or blade. First the total of the artefacts is considered and then only the complete ones and the proximal fragments, in order not to increase the sample size.

\begin{tabular}{|c|c|c|c|c|c|c|c|c|c|c|c|c|}
\hline & \multicolumn{6}{|c|}{ Total artefactos } & \multicolumn{6}{|c|}{ Instrumentos, desechos enteros y fg.prox. } \\
\hline & \multicolumn{2}{|c|}{ Grado 1} & \multicolumn{2}{|c|}{ Grado 2} & \multicolumn{2}{|c|}{ Grado 3} & \multicolumn{2}{|c|}{ Grado 1} & \multicolumn{2}{|c|}{ Grado 2} & \multicolumn{2}{|c|}{ Grado 3} \\
\hline & n & $\%$ & $\mathrm{n}$ & $\%$ & $\mathrm{n}$ & $\%$ & $\mathrm{n}$ & $\%$ & $\mathrm{n}$ & $\%$ & $\mathrm{n}$ & $\%$ \\
\hline Raederas & 0 & 0 & 1 & 0.35 & 0 & 0 & 0 & 0 & 1 & 0.52 & 0 & 0 \\
\hline RBO & 0 & 0 & 3 & 1.06 & 0 & 0 & 0 & 0 & 3 & 1.57 & 0 & 0 \\
\hline Cortantes & 0 & 0 & 1 & 0.35 & 0 & 0 & 0 & 0 & 1 & 0.52 & 0 & 0 \\
\hline Muescas & 0 & 0 & 1 & 0.35 & 0 & 0 & 0 & 0 & 1 & 0.52 & 0 & 0 \\
\hline Artef.bif. & 0 & 0 & 1 & 0.35 & 0 & 0 & 0 & 0 & 1 & 0.52 & 0 & 0 \\
\hline Inst. fragm. & 0 & 0 & 1 & 0.35 & 0 & 0 & 0 & 0 & 1 & 0.52 & 0 & 0 \\
\hline Núcleos & 0 & 0 & 9 & 3.17 & 2 & 5.13 & 0 & 0 & 8 & 4.19 & 1 & 3.33 \\
\hline Hojas & 0 & 0 & 10 & 3.52 & 1 & 2.56 & 0 & 0 & 9 & 4.71 & 1 & 3.33 \\
\hline Ls.prim. & 2 & 66.67 & 40 & 14.08 & 9 & 23.08 & 0 & 0 & 27 & 14.14 & 6 & 20 \\
\hline Ls.sec. & 0 & 0 & 22 & 7.75 & 3 & 7.69 & 0 & 0 & 16 & 8.38 & 3 & 10 \\
\hline Ls.c/dorso & 0 & 0 & 2 & 0.70 & 0 & 0 & 0 & 0 & 1 & 0.52 & 0 & 0 \\
\hline Ls.ang. & 0 & 0 & 98 & 34.51 & 13 & 33.33 & 0 & 0 & 70 & 36.65 & 11 & 36.67 \\
\hline Ls.plana & 0 & 0 & 7 & 2.46 & 1 & 2.56 & 0 & 0 & 3 & 1.57 & 1 & 3.33 \\
\hline Ls.arista & 1 & 33.33 & 68 & 23.94 & 10 & 25.64 & 0 & 0 & 46 & 24.08 & 7 & 23.33 \\
\hline Ls.flanco & 0 & 0 & 3 & 1.06 & 0 & 0 & 0 & 0 & 2 & 1.05 & 0 & 0 \\
\hline Ls.indif. & 0 & 0 & 15 & 5.28 & 0 & 0 & 0 & 0 & 1 & 0.52 & 0 & 0 \\
\hline Chunk & 0 & 0 & 2 & 0.70 & 0 & 0 & 0 & 0 & 0 & 0 & 0 & 0 \\
\hline Total & 3 & 100 & 284 & 100 & 39 & 100 & 0 & 0 & 191 & 100 & 30 & 100 \\
\hline
\end{tabular}

lascas primarias. Éstas también se han recuperado para los grados 2 y 3. Las lascas secundarias se han identificado para los grados 2 y 3 . Por otra parte, el alto número de lascas internas (lascas angulares, de arista y planas), que constituyen las mayores frecuencias de la muestra, indica que el proceso de talla continuó en el sitio. Las tendencias son similares si se considera la totalidad de los artefactos (donde constituyen el 60,91\% de los artefactos de grado 2 y el 61,53\% de los artefactos de grado 3) o únicamente aquéllos que poseen talón (enteros y fragmentos proximales) (donde constituyen el 62,21\% de los artefactos de grado 2 y el 63,33\% de los artefactos de grado 3).

En lo que respecta al paralelismo de las extracciones, condición necesaria para desarrollar un desbaste laminar, se han recuperado hojas y lascas de arista tanto en los casos del grado 2 como en el del 3. Si bien esto indica que era una de las formas base que se buscaba obtener, esto no implicó que el desbaste se orientara a obtener artefactos laminares. Las hojas constituyen el 3,52\% del total de los artefactos del grado 2 , y el $4,71 \%$ si se consideran únicamente los ejemplares con talón. En el caso del grado 3, alcanzan únicamente el 2,56\% del total de los artefactos y el 3,33\% de los casos con talón. La tendencia aumenta entre las lascas de arista. En madera del grado 2 alcanzan el 23,94\% si se considera el total de artefactos y el $24,08 \%$ si se tienen en cuenta sólo los artefactos con talón. En el caso del grado 3 alcanzan el $25,64 \%$ para la totalidad de la muestra y el $23,33 \%$ si se consideran únicamente los artefactos con talón. No debe descartarse, sin embargo, que algunos de estos ejemplares se trasladen a espacios cercanos, donde se encuentren otros tipos de sitios.

El hallazgo de un percutor duro entero próximo a los troncos silicificados $(40 \mathrm{~m})$ podría deberse a que se depositó en el sitio por razones de transportabilidad (sensu Nelson 1991), dado su peso y por la posibilidad de regresar al sitio en momentos diferidos, aunque no puede descartarse totalmente que haya sido abandonado por la posibilidad de ser reemplazado de manera relativamente sencilla Esto deberá ser analizado con muestreos específicos destinados a evaluar la disponibilidad de materias primas adecuadas para ser utilizadas como percutores en el área.

En lo que se refiere a los núcleos, dentro del grado 2 predominan los prismáticos (4 ejemplares: $66,67 \%$ de la muestra), habiéndose identificado también discoidales y poliédricos (1 en cada caso) (Figura 3). Únicamente se recuperaron dos ejemplares del grado 3, que son prismáticos. La baja frecuencia de núcleos con alto grado de silicificación sugiere también su traslado a espacios próximos. La mayor parte de los núcleos de grado 2 presentan más de un $50 \%$ de corteza. Con una única excepción, están enteros. Seis de ellos presentan extracciones paralelas a las vetas de la madera silicificada, mientras que en tres casos son transversales. Los núcleos de grado 3 presentan extracciones transversales, lo que es consistente con lo observado en el caso de los desechos. 


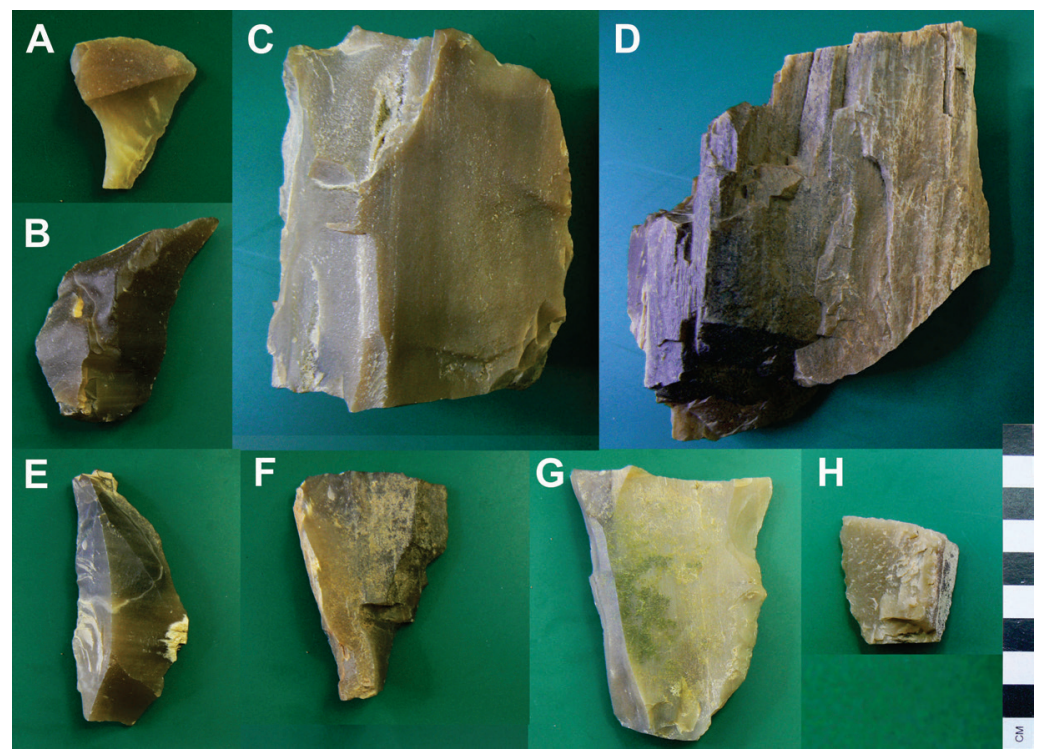

Figura 3. Artefactos recolectados en los relevamientos. Referencias: A. Lasca angular; B: Lasca primaria; C: Núcleo prismático irregular; D. Núcleo poliédrico; E. Lasca de arista; F. RBO; G. RBO; H. Raedera.

Figure 3. Artefacts collected during the surveys. References: A: Multidirectional flake; B: Cortical flake; C: Irregular prismatic core; D: Polyhedral core; E: Bladelike flake; F: Small side-scraper; G: Small side-scraper; H: Side-scrapper.

\section{Consideraciones generales}

Los análisis realizados permitieron observar un aprovechamiento diferencial de los troncos en relación con el grado de silicificación y, en consecuencia, con la calidad de la materia prima. En este sentido, no se han identificado artefactos de grado 0 , mientras que es mínima la presencia de ejemplares de grado 1.

Por otra parte, las extracciones no están condicionadas por el grado de silicificación en el caso de las materias primas de mejor calidad (grado 3). En el caso del grado 2 se observan tanto extracciones paralelas como transversales a las vetas, por lo que la situación es variable, aunque de cualquier manera existe una alta frecuencia de extracciones transversales. Lo observado en los desechos es consistente con los resultados obtenidos a partir de los núcleos. La baja frecuencia de núcleos y la presencia de múltiples extracciones en los troncos sugieren que el desbaste no se basó tanto en la formatización de núcleos, sino en la extracción de lascas o lascas nodulares. No puede descartarse, sin embargo, que esta diferencia se deba al transporte hacia espacios próximos.

La menor cantidad de desechos de grado 3 podría deberse a su menor disponibilidad en la fuente, a lo que podría sumarse su traslado a otros sectores, vinculado a su mejor calidad. En este sentido, la baja frecuencia de instrumentos también podría relacionarse con su traslado hacia otros sectores del espacio. El transporte también podría explicar la baja frecuencia de núcleos con alto grado de silicificación. Todo esto apuntaría hacia una selectividad de los artefactos que se transportan.

El hallazgo de un percutor duro entero sugiere que fue dejado en el lugar para su utilización futura, lo que implicaría un equipamiento del sitio (sensu Binford 1979). Este tipo de estrategia puede relacionarse con el hecho de que en el área no abundan las fuentes primarias de madera silicificada, por lo que esta fuente podría revestir cierta relevancia para los grupos cazadores-recolectores

Por otra parte, debe recordarse que en general, los percutores son muy valorados por los talladores, lo que apuntaría a sostener la idea del retorno al sitio. Su abandono también podría relacionarse con priorizar el traslado de los artefactos obtenidos en la cantera hacia otros sitios, por razones de transportabilidad. Otra posibilidad es que sea posible el reemplazo relativamente sencillo de los percutores, lo que, deberá ser evaluado en el futuro en relación con muestreos específicos.

Como se mencionó en el apartado Antecedentes, durante el Holoceno medio se registra en el extremo sur del Macizo del Deseado un aumento en la laminaridad. Dado que la madera silicificada de grado 2 o 3 no presenta restricciones relativas al método de desbaste, su utilización para el desbaste laminar (que involucra volúmenes prismáticos y formas base guiadas por aristas) no responde a una restricción técnica de la materia prima, sino que se debe a una elección técnica que deberá ser evaluada en cada caso particular.

Buenos Aires, 9 de agosto de 2019.

\section{Agradecimientos}

Esta investigación fue financiada con fondos del proyecto PICT 2015-2038. Nuestro agradecimiento a los organizadores del simposio en el que originalmente se presentó este trabajo, a la Dirección de Patrimonio Cultural de la Provincia de Santa Cruz, a la Dirección de Cultura y Turismo de Gobernador Gregores, al Sr. Juan Pablo Sayes (Ea. El Verano), las Sras. María Díaz y Florence Kemp (Ea. 17 de Marzo) y a las empresas y personal de Piedra Grande S.A. y Minera Triton S.A., especialmente al Dr. Claudio Iglesias y al Lic. Ricardo Silva. A dos evaluadores anónimos, cuyos comentarios ayudaron a 
mejorar este manuscrito. A Brenda Gilio y Bruno Pollard, quienes participaron en los trabajos de campo.

\section{Referencias citadas.}

Aguerre, A.M. (2003). La Martita: ocupaciones de 8000 años en la Cueva 4. En A. Aguerre (Ed.) Arqueología y Paleoambiente en la Patagonia Santacruceña Argentina (29-61). Ediciones del Autor, Buenos Aires.

Andrefsky Jr., W. (1994). Raw material availability and the organization of technology. American Antiquity 59 (1), 21-34. Society for American Archaeology.

Aschero, C.A. (1975). Ensayo para una clasificación morfológica de artefactos líticos aplicada a estudios tipológicos comparativos. Informe al CONICET. MS. Revisión 1983.

Aschero, C.A 1983. Ensayo para una clasificación morfológica de artefactos líticos aplicada a estudios tipológicos comparativos. Informe al CONICET. MS.

Binford, L. (1979). Organization and formation processes: looking at curated technologies. Journal of Anthropological Research 35 (255-273).

Brook, G.A., M.V. Mancini, N.V. Franco, F. Bamonte y P. Ambrústolo. (2013). An examination of possible relationships between paleoenvironmental conditions during the Pleistocene-Holocene transition and human occupation of southern Patagonia (Argentina) east of the Andes, between $46^{\circ}$ and $52^{\circ} \mathrm{S}$. Quaternary International 305 (104-118).

Callahan, E. (1979). The basics of biface knapping in the eastern fluted point tradition: a manual for flintknappers and lithic analysts. Archaeology of Eastern North America 7 (1), 1-180. Washington, D.C.

Cattáneo, R. (2000). El paisaje y la distribución de recursos líticos en el Nesocratón del Deseado. En L. Miotti, R. Paunero, M. Salemme y R. Cattaneo (Eds.) Guía de Campo de la visita a las localidades arqueológicas, Taller Internacional del INQUA: La Colonización del Sur de América durante la transición Pleistoceno-Holoceno (26-35). La Plata.

Cattáneo, R. (2004). Desarrollo metodológico para el estudio de fuentes de aprovisionamiento lítico en la Meseta Central Santacruceña, Patagonia Argentina. Estudios Atacameños 28 (105-119).

Cattáneo, R. (2005a). Tecnología lítica del Pleistoceno Final/Holoceno Medio. Un estudio de los cazadoresrecolectores de la Patagonia Austral (Argentina). BAR International Series.
Cattáneo, R. (2005b). Tecnología lítica en la localidad arqueológica Piedra Museo (Santa Cruz, Argentina). Relaciones de la Sociedad Argentina de Antropología 30 (79-103).

Crabtree, D.E. (1972). An Introduction to Flintworking. Occasional Papers of the Idaho State University Museum 28. Idaho State University Museum.

Durán, V., A. Gil, G. Neme y A. Gasco. (2003). El Verano: ocupaciones de 8900 años en la Cueva 1 (Santa Cruz, Argentina). En A. Aguerre (Ed.) Arqueología y Paleoambiente en la Patagonia Santacruceña Argentina (93-120). Ediciones del autor, Buenos Aires.

Echeveste, H. (2005). Travertinos y jasperoides de Manantial Espejo, un ambiente Hot Spring Jurásico. Macizo del Deseado, Provincia de Santa Cruz, Argentina. Latin American Journal Sedimentology and Basin Analysis 12 (1), 33-48.

Eren, M., C.I. Roos, B.A. Story, N. von Craamon-Taubadel y S.J. Lycett. (2014). The role of raw material differences in stone tool shape variation: an experimental assessment. Journal of Archaeological Science 48 (472-487).

Franco, N.V., P. Ambrústolo, M. Martucci, G.A. Brook, M.V. Mancini, y N. Cirigliano. (2010). Early Human Occupation in the Southern Part of the Deseado Massif (Patagonia, Argentina). Current Research in the Pleistocene 27 $(13-16)$.

Franco, N.V., Pablo Ambrústolo, A. Acevedo, N. Cirigliano, y M. Vommaro. (2013). Prospecciones en el sur del Macizo del Deseado (provincia de Santa Cruz). Los casos de La Gruta y Viuda Quenzana. En A.F. Zangrando, R. Barberena, A. Gil, G. Neme, M. Giardina, L. Luna, C. Otaola, S. Paulides, L. Salgán, y A. Tívoli (Eds.) Tendencias teóricometodológicas y casos de estudio en la Arqueología de la Patagonia (371-378). Museo de Historia Natural de San Rafael. Altuna Impresores, Buenos Aires.

Franco, N.V., P. Ambrústolo y L. Vetrisano. (2015). Materias primas líticas y su utilización en las cuencas de los ríos Chico y Santa Cruz (provincia de Santa Cruz, Patagonia argentina). Intersecciones en Antropología 16 (Sup. 1), 113-123.

Franco, N.V., G.A. Brook, M.V. Mancini y L. Vetrisano. (2016). Changes in lithic technology and environment in southern continental Patagonia: The Chico and Santa Cruz River basins. Quaternary International Volume 422 (57-65).

Franco, N.V., L.A. Borrero y G. Lucero. (2019). Human dispersal in the Atlantic slope of Patagonia and the role of lithic availability. Paleoamérica. A journal of early human migration and dispersal. DOI: 10.1080/20555563.2019.1582128 
Frank, A.D., F. Skarbun y M. Cueto. (2013). Caracterización de una fuente de xilópalo: el Bosque Petrificado de la localidad Arqueológica La María. En A. F. Zangrando, R. Barberena, A. Gil, G. Neme, M. Giardina, L. Luna, C. Otaola, S. Paulides, L. Salgán y A. Tívoli (Eds.) Tendencias teórico-metodológicas y casos de estudio en la Arqueología de la Patagonia (379-388). Museo de Historia Natural de San Rafael. Altuna Impresores, Buenos Aires.

Gradin, C.J., C.A. Aschero, A.M. Aguerre. (1979). Arqueología del Área Río Pinturas (Provincia de Santa Cruz). Relaciones de la Sociedad Argentina de Antropología XIII (183-227).

Hermo, D. (2005). Fuentes de aprovisionamiento de xilópalo en el Monumento Natural Bosque Petrificado, provincia de Santa Cruz. Entre Pasados y Presentes. Trabajos de las VI Jornadas de Jóvenes Investigadores en Ciencias Antropológicas (235-246). INAPL. Buenos Aires.

Hermo, D. (2008). Los cambios en la circulación de las materias primas líticas en ambientes mesetarios de Patagonia. Una aproximación para la construcción de los paisajes arqueológicos de las sociedades cazadorasrecolectoras. Tesis doctoral inédita. Universidad Nacional de La Plata, La Plata.

Inizan, M-L; H. Roche y J. Tixier. (1992). Technology of Knapped Stone. Meudon, CREP.

Manninen, M. y K. Knutsson. (2014). Lithic raw material diversification as an adaptive strategy - Technology, mobility, and site structure in Late Mesolihic northernmost Europe. En Journal of Anthropological Archaeology 33 (84-98).

Miotti, L. y R. Cattaneo. (2003). Variations in the Strategies of Lithic Production and Faunal Exploitation during the Pleistocene/Holocene Transition at Piedra Museo and Surrounding Region. En L. Miotti, M. Salemme y N. Flegenheimer (Eds.) Where the South Winds Blow: Ancient Evidences of Paleo South Americans (105-111). Center for the Studies of the First Americans, Texas A\&M University.

Mosquera, B. (2016). Geoarqueología de la cuenca de los zanjones Blanco y Rojo, Macizo del Deseado. Tesis doctoral inédita. Universidad Nacional de La Plata, La Plata.

Nami, H. (1986). Experimentos para el estudio de la tecnología bifacial de las ocupaciones tardías en el extremo sur de la Patagonia Continental. En Informes de Investigación 5, PREPS, CONICET. Buenos Aires.
Nelson, M.C. (1991). The Study of Technological Organization. En M. Schiffer (Ed.) Archaeological Method and Theory Vol. 3 (57-100). University of Arizona Press, Tucson.

Odell, G.H. (2004). Lithic Analysis. Kluwer Academic/ Plenum Publishers, New York.

Panza, J.L. y G. Marin. (1998). Geología. Hoja Geológica 4969-I "Gobernador Gregores", Provincia de Santa Cruz. Boletín 239, (1-104). SEGEMAR, Buenos Aires.

Panza, J.L. y M. J. Haller. (2002). El vulcanismo Jurásico. Geología y Recursos Naturales de Santa Cruz. En M. Haller (Ed.) Relatorio del XV Congreso Geológico Argentino, Actas I (89-101). Asociación Geológica Argentina, Buenos Aires.

Paunero, R.S. (2000). Localidad arqueológica Cerro Tres Tetas. En L. Miotti, R. Paunero, M. Salemme, y R. Cattáneo (Eds.). Guía de Campo de la visita a las localidades arqueológicas, (89-100). INQUA International Workshop "La Colonización del Sur de América durante la transición Pleistoceno/Holoceno". La Plata.

Paunero, R.S. (2003). The Cerro Tres Tetas (C3T) Locality in the Central Plateau of Santa Cruz, Argentina. En L. Miotti, M. Salemme and N. Flegenheimer (Eds.) In Where the South Winds Blow: Ancient Evidences of Paleo South Americans (133-140). Center for the Studies of the First Americans, Texas A\&M University.

Ramos, V.A. (1999). Las provincias geológicas del territorio argentino. Geología Argentina. Anales 29 (3), 41-96.

Skarbun, F. (2009). La organización tecnológica en grupos cazadores-recolectores desde las ocupaciones del Pleistoceno final al Holoceno Tardío en la Meseta Central de Santa Cruz. Tesis doctoral inédita. Universidad Nacional de La Plata, La Plata, Argentina.

Skarbun, F., A. Frank, M. Paunero, M. Cueto y G. Rosales. (2007). Análisis de la tecnología lítica del sitio Casa del Minero 1, Meseta Central de Santa Cruz. En F. Morello, A. Prieto, M. Martinic y C. Bahamonde (Eds.) Arqueología de Fuego-Patagonia. Levantando Piedras, Desenterrando Huesos... y Develando Arcanos (589-600). CEIDER, Punta Arenas.

Whittaker, J. (1994). Making and Understanding Stone Tools. University of Texas Press, Texas.

Zubia, M. 1998. Recursos Minerales. Hoja Geológica 4969-I "Gobernador Gregores", Provincia de Santa Cruz. Boletín 239, (75-80). SEGEMAR. Buenos Aires. 